\title{
Computational Analysis of Different Artificial Intelligence Based Optimization Techniques for Optimal Power Flow and Economic Load Dispatch Problem
}

\author{
Debirupa Hore \\ Department of Electrical Engg. \\ K J Educational Institutes, KJCOEMR, Pune \\ E-mail : debirupa1904@gmail.com
}

\author{
N M Lokhande \\ Department of Electrical Engg. \\ K J Educational Institutes, KJCOEMR, Pune \\ E-mail : netralokhande@gmail.com
}

\begin{abstract}
The purpose of this paper is to present a computational Analysis of various Artificial Intelligence based optimization Techniques used to solve OPF problems. The various Artificial Intelligence methods such as GA, PSO, BFO, ANN are studied and analyzed in detail. The objective of an Optimal Power Flow (OPF) algorithm is to find steady state operation point which minimizes generation cost and transmission loss etc. or maximizes social welfare, load ability etc. while maintaining an acceptable system performance in terms of limits on generators' real and reactive powers, power flow limits, output of various compensating devices etc. Traditionally, Classical optimization methods were used effectively to solve optimal power flow. But, recently due to the incorporation of FACTS devices and deregulation of power sector the traditional concepts and practices of power systems are superimposed by an economic market management and hence OPF have become more complex. So, in recent years, Artificial Intelligence (AI) methods have been emerged which can solve highly complex OPF problems at faster rate.
\end{abstract}

\section{INTRODUCTION}

Growing demand of the power and complexity of the power system network, power system study has become a significant tool for a power system operator in order to take corrective actions in time. Besides giving real and reactive power the load flow study provides information about line and transformer loading as well as losses throughout the system and also voltages at different points in the system for evaluation and regulation of the performance of the power systems. Further study and analysis of future expansion, stability and reliability of the power system network can be easily analyzed through this study. We can define the main objectives of OPF as to minimize the cost of generation simultaneously satisfying all the security constraints as well as to minimize the transmission losses. Optimal Power Flow problem is a highly non linear problem hence different conventional optimization methods where developed for load flow solutions. The popular traditional 'Gauss- Siedel' iterative method which require minimal computer storage through Y-matrix. Although the performance is satisfactory on different systems but the main drawback is its converging time. To overcome this deficiency led to the development of $\mathrm{Z}$ matrix methods, which converge more reliably but sacrifice some of the advantages of Y-matrix iterative methods notably storage and speed when applied to large systems. The other conventional methods like Newton-Raphson method was shown to have powerful convergence properties, but was computationally uncompetitive and the contribution is restricted to the problem of convergence of the solution.

Currently, with the stimulus of increasing problem sizes, online applications, and system optimization, newer methods are emerging which are also expected to find wide applications. For review, a balanced three phase power system along with transmission line has been considered. The universally preferred network analysis nodal admittance matrix equation is used and is as shown below.

$$
\mathrm{I}=\mathrm{Y} * \mathrm{E}
$$

Where, matrix $\mathrm{Y}$ is square, sparse, and symmetrical (in the absence of phase shifters or mutual couplings represented by non-bilateral network branches).With the increase of the Complexity and non linearity of the power system different Conventional optimization technique has emerged such as $\mathrm{Y}$ matrix Iterative Load Flow Methods, Z-matrix Load Flow Methods, Newton Raphson Method, Fast Decoupled Method etc. It has also been noted the OPF problem with series compensation may be a non Convex and non linear problem which leads to Conventional optimization methods stuck to local minimum.

The computational intelligence algorithms of different non conventional optimization techniques for OPF such as Particle Swarm Optimization, Genetic Algorithm, Bacterial Forging and Artificial Neural Network are the recent emerging trends in the power system analysis. The paper presents the detail analysis of different above techniques.

\section{LOAD FLOW REVIEW}

The prime function of an Electric Power System is to supply the power demand in an efficient, economic, high quality and reliable way. The power system can operate in an infinite number of states - voltage and power sets in the buses - in order to comply with standard requisites.

The solution of a Power Flow problem consists in the determination of these possible operational states through the knowledge a priori of certain variables of the system buses. The objective of this kind of problem is to obtain the system buses voltages - module and angle - in order to determine later the power adjustments in the generation buses and the power flow in the system lines. The power flow study provides the system status in the steady-state, i.e., its parameters do not vary with the time variation. 
The importance of the power flow calculation is also the obtaining of an optimal point of operation, regarding to quality and economy. Once the steady-state of the system is known, it is possible to estimate the amount of power generation necessary to supply the power demand plus the power losses in the system lines, moreover the voltage levels must be kept within the boundaries and overloaded operations, besides the operations in the stability limit must be avoided.

The general form of the Static Load Flow Equations (SLFE) is given by equation (1)

$P_{i}-j Q_{i}-y_{i 1} V_{1} V_{i}^{*}-y_{i 2} V_{2} V_{i}^{*}-y_{i n} V_{n} V_{i}^{*}=0$

Where, $i=1 \ldots \ldots n$, bus number; $P_{i}=$ active power generated or injected in the bus $i ; Q_{i}=$ reactive power generated or injected in the bus $\mathrm{i} ;\left|\mathrm{V}_{\mathrm{i}}\right|=$ voltage module of the bus $\mathrm{i} ; \delta_{\mathrm{i}}=$ voltage angle of the bus $\mathrm{i} ; \mathrm{V}_{\mathrm{i}}=\left|\mathrm{V}_{\mathrm{i}}\right| \mathrm{e}^{\mathrm{j} \delta \mathrm{i}}$, i.e., the voltage in the polar form; $\mathrm{y}_{\mathrm{ik}}=$ element of the nodal admittance matrix $\mathrm{Y}_{\text {bus. }}$. The nodal admittance matrix is obtained through the following explanation: if $\mathrm{i}=\mathrm{k}, \mathrm{y}_{\mathrm{ik}}$ is the sum of the admittances that come out of the bus $i$; and if $i \neq k, y_{i k}$ is the admittance between the buses $i$ and $k$, multiplied by -1 .

The power system buses are classified in types, according to the variables known a priori and to the variables that will be obtained through the SLFE.

- Type 1 Bus or PQ Bus: $\mathrm{P}_{\mathrm{i}}$ e $\mathrm{Q}_{\mathrm{i}}$ are specified and $\left|\mathrm{V}_{\mathrm{i}}\right|$ and $\delta_{\mathrm{i}}$ are obtained through the SLFE;

- Type 2 Bus or PV Bus: $P_{i}$ e $\left|V_{i}\right|$ are specified and $Q_{i}$ and $\delta_{i}$ are obtained through the SLFE;

- Type 3 Bus or V $\delta$ Bus ("Slack Bus"): $\left|\mathrm{V}_{\mathrm{i}}\right|$ and $\delta_{\mathrm{i}}$ are specified and $P_{i}$ and $Q_{i}$ are obtained through the SLFE.

Equation (1) performs a complex and non-linear equations system, and its solution is obtained through approximations using numeric computational methods. The methods Gauss, Gauss-Seidel and Newton- Raphson are often applied to solve these systems. Newton-Raphson is the most used due to its greater guarantee and speed of convergence.

The methods consist in the adoption of initial estimated values to the bus voltages, module 1,0 [p.u] and angle $0[\mathrm{rad}]$, for instance, and in the application of the SLFE in successive iterations, searching for better approximations for the voltages. The stop criterion varies according to the required accuracy.

\section{ECONOMIC LOAD DISPATCH}

Economic Load Dispatch (ELD) problem is one of the fundamental issues in power system. It is an optimization problem and its objective is to reduce the total power generation cost of units while satisfying load demands and load constraints. Economic dispatch is a process of allocating generation levels to the generating units so that the system load is fully supplied in the most economic way and it is defined as the operation of generation facilities to produce energy at lowest cost to reliably serve consumers, recognizing any operational limits of generation and transmission facilities. The primary objective of the Economic Dispatch(ED) problem is to determine the most economic loadings of the generators such that the load demand in a power system can be met. Previous conventional methods for solving ELD problem are based on mathematical techniques. For highly non linear and combinatorial optimization problems, the conventional methods are facing difficulties to locate the global optimal solution. Recently there is an upsurge in the use of modern soft computing in the arena of power system optimization techniques.

\section{PROBLEM FORMULATION}

The objective of the Economic Load Dispatch (ELD) problem is to minimize the total fuel cost of thermal power plants subjected to the operating constraints of a power system. The thermal power plant can be expressed as input-output models(i.e cost function), where the input is the fuel cost and the output the power output of each unit, in practice, operating cost of each generator is represented by a quadratic function. The cost depends on the number of hours the plant is in operation or upon the number of units of electrical energy generated i.e the operating cost is approximately proportional to power units generated is given by

Total Cost $=\mathrm{C}_{\mathrm{t}}=\sum \mathrm{F}_{\mathrm{i}}\left(\mathrm{P}_{\mathrm{i}}\right)$ $i=1$

The fuel cost functions of the plant generating units are generally characterized by second order polynomials given as equation (3).

$$
F_{i}\left(P_{i}\right)=\alpha_{i}+\beta_{i} P_{i}+\gamma_{i} P_{i}^{2}
$$

Where

$\mathrm{P}_{\mathrm{i}}$ - Output power generation of ith unit.

$\alpha_{i}, \beta_{i}, \gamma_{i}-$ Fuel cost coefficients of $i^{\text {th }}$ unit.

Some other constraints which is as

\section{Power Balance Constraints}

This constraint is based on the principle of equilibrium between the total system generation and the total system loads( PD) and losses (PL) which must be equal; also the cost function is not affected by the reactive power demand. Hence the power system balance equation is as

$$
\sum_{i=1}^{n_{g}} P_{i}-\left(P_{D}+P_{L}\right)=0
$$

\section{The Generator Constraints}

The maximum active power generation $(\mathrm{Pi})$ is limited by thermal considerations and also minimum power generation is limited by the flame instability of a boiler. If the power output of a generator for optimum operation of the system is less than a prespecified value, the unit is not to put on the bus bar because it is not possible to generate that low value of power from the unit. So the output power of each power generating unit has a lower and upper bound and unit power lies in between these bounds.

$$
\mathrm{P}_{\text {imin }} \leq \mathrm{P}_{\mathrm{i}} \leq \mathrm{P}_{\text {imax }}
$$

Where $\mathrm{P}_{\text {imin }}$ and $\mathrm{P}_{\text {imax }}$ denote the minimum and maximum output power generation respectively of $i^{\text {th }}$ unit. 
The total transmission network loss is quadratic function of power outputs that can be represented using B coefficients.

$$
\mathrm{P}_{\mathrm{L}}=\sum_{\mathrm{i}=1 \mathrm{j}=1}^{\mathrm{n}_{\mathrm{g}} \mathrm{P}_{\mathrm{i}} \sum_{\mathrm{i}} \mathrm{P}_{\mathrm{i}} \mathrm{B}_{\mathrm{ij}} \mathrm{P}_{\mathrm{j}}}
$$

where $B_{i j}$ are loss coefficients, which are assumed to be constant.

Following is the study of different Artificial Intelligence Techniques .

\section{GENETIC ALGORITHM}

Genetic algorithms [1] are search algorithms based on the process of biological evolution. In genetic algorithms, the mechanics of natural selection and genetics are emulated artificially. The search for a global optimum to an optimization problem is conducted by moving from an old population of individuals to a new population using genetics-like operators. Each individual represents a candidate to the optimization solution. An individual is modeled as a fixed length string of symbols, usually taken from the binary alphabet. An evaluation function, call fitness function, assigns a fitness value to each individual within the population. This fitness value is measure for the quality of an individual. The basic optimization procedure involves nothing more than processing highly fit individuals in order to produce better individuals as the search progresses. A typical genetic algorithm cycle involves four major processes of fitness evaluation, selection, recombination and creation of a new population. Although the binary representation is usually applied to power optimization problems, in this paper, we use the real valued representation scheme for solution. The use of real valued representation in the GA is claimed by Wright to offer a number of advantages in numerical function optimization over binary encoding. Efficiency of the GA is increased as there is no need to convert chromosomes to the binary type; less memory is required as efficient floating-point internal computer representations can be used directly; there is no loss in precision by discretisation to binary or other values; and there is greater freedom to use different genetic operators. For the real valued representation, the k-th chromosome

$C_{K}$ can be defined as follows

$C_{K}=\left[P_{K 1}, P_{K 2} \ldots \ldots \ldots P^{P_{i]}}\right] \mathrm{K}=1.2 \ldots \ldots \ldots$ pop size

Where pop size means population size and $P_{\mathrm{KI}}$ is the generation power of the i-th unit at k-th chromosome.

Reproduction involves creation of new offspring from the mating of two selected parents or mating pairs. It is thought that the crossover operator is mainly responsible for the global search property of the GA. We used an arithmetic crossover operator that defines a linear combination of two chromosomes.

Two chromosomes, selected randomly for crossover, $C_{\mathrm{i}}^{g e n}$ and

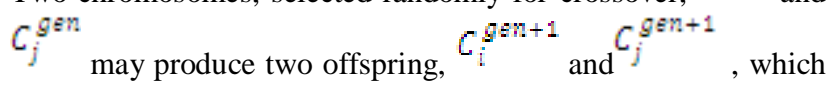
is a linear combination of their parents i.e.,

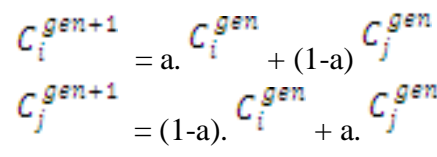

Where $\mathrm{a}$ is a random number in range of $[0,1]$.

The mutation operator is used to inject new genetic material into the population and it is applied to each new structure individually. A given mutation involves randomly altering each gene with a small probability. We generate a random real value which makes a random change in the m-th element selected randomly of the chromosome. The objective function [11] is used to provide a measure of how individuals have performed in the problem domain. In the case of a minimization problem, the fit individuals will have the lowest value of the associated objective function. The fitness function is normally used to transform the objective function value into a measure of relative fitness. The fitness function is defined as

$\operatorname{Fit}(\mathrm{x})=\mathrm{g}(\mathrm{f}(\mathrm{x}))$

where $f(x)$ is the objective function, $g$ transforms the value of the objective function to non-negative number.

An elitist which GA search is used guarantees that the best solution so far obtained in the search is retained and used in the following generation, $\mathrm{n}$ and thereby ensuring no good solution already found ean be lost in search process.

\section{BACTERIAL FORAGING OPTIMIZATION ALGORITHM}

This procedure called foraging is crucial in natural selection, since the animals with poor foraging strategies are eliminated, and successful ones tend to propagate. Hence, to survive, an animal or a group of animals must develop an optimal foraging policy. Some of the most successful foragers are bacteria like the E.Coli, which employs chemical sensing organs to detect the concentration of nutritive or noxious substances in its environment. The bacteria then moves through the environment by a series of tumbles and runs, avoiding the noxious substances and getting closer to food patch areas in a process called Chemotaxis. Besides, the bacteria can secrete a chemical agent that attracts its peers, resulting in an indirect form of communication.

The foraging strategy is governed basically by four processes namely Chemotaxis, Swarming, Reproduction, Elimination and Dispersal

\section{a. Chemotaxis}

Chemotaxis process is the characteristics of movement of bacteria in search of food and consists of two processes namely swimming and tumbling. A bacterium is said to be 'swimming' if it moves in a predefined direction, and 'tumbling' if moving in an altogether different direction. Let $\mathrm{j}$ be the index of Chemo tactic step, $\mathrm{k}$ be the reproduction step and $\mathrm{l}$ be the elimination dispersal event. Let is the position of ith bacteria at jth chemo tactic step, $k$ th reproduction step and th elimination dispersal event. The position of the bacteria in the next chemo tactic step after a tumble is given by-

$$
\theta^{i}(j+1, k, l)=\theta^{i}(j, k, l)+C(i) \cdot \frac{\Delta(i)}{\sqrt{\Delta^{T}(i)}}
$$


If the health of the bacteria improves after the tumble, the bacteria will continue to swim to the same direction for the specified steps or until the health degrades.

\section{b. Swarming}

Bacteria exhibits swarm behavior i.e. healthy bacteria try to attract other bacteria so that together they reach the desired location (solution point) more rapidly. The effect of Swarming is to make the bacteria congregate into groups and move as concentric patterns with high bacterial density. Mathematically swarming behavior can be modeled as

$$
\begin{aligned}
& J_{c e}\left(\Theta_{s} p\left(j, k_{s} D\right)=\sum_{i=1}^{n z} J_{c e}\left(\theta_{v} \theta^{i}\left(j, k_{s} D\right)\right.\right. \\
& =\sum_{i=1}^{S}\left[-d_{\text {attract }} \exp \left(-W_{\text {attract }} \sum_{i=1}\left(\theta_{m}-\theta_{m}^{i}\right)_{2}\right)\right]
\end{aligned}
$$

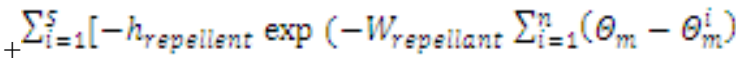

\section{c. Reproduction}

In this step, population members who have had sufficient nutrients will reproduce and the least healthy bacteria will die. The healthier half of the population replaces with the other half of bacteria which gets eliminated, owing to their poorer foraging abilities. This makes the population of bacteria constant in the evolution process.

\section{d. Elimination and Dispersal}

In the evolution process a sudden unforeseen event may drastically alter the evolution and may cause the elimination and/or dispersion to a new environment. Elimination and dispersal helps in reducing the behavior of stagnation i.e. being trapped in a premature solution point or local optima.

\section{PARTICLE SWARM OPTIMIZATION}

In 1995, Kennedy and Eberhart first introduced the PSO method, motivated with the social behavior of organisms such as fish schooling and bird flocking. PSO, as an optimization tool, provides a population based search procedure in which individuals called particles change their positions (states) with time. In a PSO system, particles fly around in a multidimensional search space. During flight, each particle adjusts its position according to its own experience, and the experience of neighboring particles, making use of the best position encountered by itself and its neighbors. The swarm direction of a particle is defined by the set of particles neighboring the particle and its history experience.

Let $\mathrm{x}$ and $\mathrm{v}$ denote a particle coordinates (position) and its corresponding flight speed (velocity) in a search space, respectively. Therefore, the ith particle is represented as $x i=(x i 1+x i 2 \ldots \ldots x i d)$ in the d dimensional space. The best previous position of the ith particle is recorded and represented as

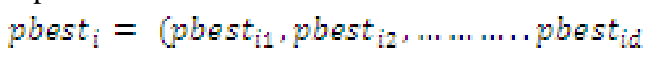

The index of the best particle among all the particles in the group is represented by $g$ best $t_{\mathbb{L}}$ the rate of the velocity for particle $\mathrm{i}$ is represented as $v i=\left(v i{ }^{i}, v i 2, \ldots x\right.$ vid $)$.
The modified velocity and position of each particle can be calculated using the current velocity in distance from pbest id to gbest $_{\mathbb{d}}$

$V_{i d}^{(t+1)}=w \cdot V_{i d}^{t}+C_{1} *$ rand $0 *\left(p\right.$ best $\left.{ }_{i d}-X_{\text {id }}^{t}\right)+$ $C_{2} *$ rand $O *\left(g\right.$ best $\left.i d-H_{i d}^{t}\right)$

$X_{i d}^{t+1}=X_{i d}^{t}+V_{i d}^{t+1}$

$\mathrm{I}=1,2 \ldots \ldots \mathrm{n} \quad \mathrm{d}=1,2 \ldots \ldots \mathrm{m}$

Where: $\mathrm{n}$ is the number of particles in a group. $\mathrm{m}$ is the number of members in a particle. $t$ is the pointer of iterations. $w$ is the inertia weight factor. $C_{1}$ and $C_{\text {2are two uniform random values }}$ in the range $[0,1]$.

is the velocity of the particle $i$ at iteration $t$

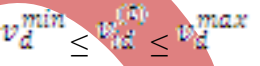

$X_{0}^{(t)}$

$X_{\mathbb{d}}$ is the current position of particle $i$ at iteration $t$. in the above procedures, the parameter $V^{\max }$ determines the resolution, or fitness, with which regions are to be searched between the present position and the target position. If $V^{\max }$ is too high, particles might fly past good solutions. If $V^{\max }$ is too small, particles may not explore sufficiently beyond local solutions. In many experiences with PSO, ${ }^{\max }$ was often set at $10-20 \%$ of the dynamic range of the variable on each dimension. The constants $C_{1}$ and $C_{2}$ represent the weighing of the stochastic acceleration terms that pull each particle toward the pbest and gbest positions. Low values allow particles to roam far from the target regions before being tugged back. On the other hand, high values result in abrupt movement towards, or past, target regions. Hence the acceleration constants $C_{1 \text { and }} C_{2}$ were often set to be 2.0 according to past experiences. Suitable selection of inertia weight $\mathrm{w}$ provides a balance between global and local explorations, thus requiring less iteration on average to find a sufficiently optimal solution. As originally developed, W often decreases linearly from about 0.9 to 0.4 during a run.

In general, the inertia weight $\mathrm{w}$ is set according to the following equation:

$W=W_{\max } * \frac{\left[W_{\max }-W_{\min } \rrbracket\right.}{\text { itammax }} *$ iter

where: $W_{\max }$ is the initial weight, $W_{\min }$ is the final weight, iter is the current iteration number, and itermax is the maximum iteration number.

\section{APPLICATION STUDY}

Rajesh K.S and S. Lyla et al. [1] develops a simple genetic algorithm methodology for secure operation of the system algorithm to 14 bus model system and the 30 bus model system and found that it is better to the other Conventional optimum dispatch problem. The technique of Genetic Algorithm proves to be powerful and practical tool for obtaining an approximate solution of a liberalized optimization problem. Also the computational time required for the proposed method was very 
less when compared to the linear programming method. The New GA technique and approach gives better result in terms of cost optimization compared to conventional Linear Programming.

Singh and Verma et al [2] proposes an application of genetic algorithm and particle swarm optimization to solve the economic dispatch problem. The transmission losses are included by calculating the $\mathrm{B}$ coefficients of transmission losses. The results were taken on 26 bus to test the effectiveness of the proposed method. The simulation result shows that there is effectively decrease in the total generation cost of the optimal dispatch solution using Genetic Algorithm and particle swarm optimization.

Kwang Y.Lee et al [3] summarize the application of PSO for solving Economic dispatch problem including the advantages and disadvantages of PSO approach. The PSO algorithm is proves to be one of the advance and faster method for solving optimization problem. Especially PSO based application to non smooth ED problems outperforms other mathematical algorithm. However PSO algorithm still needs further research and development to improve its performance and to obtain the robustness.

Djillani BEN et al [4] presents a Particle Swarm Optimization (PSO) based algorithm for optimal flow with generating units having non-smooth fuel costs curves while satisfying the constraints such as generator capacity limits. The effectiveness of the proposed algorithm was been tested on the IEEE 30-bus system with different fuel cost characteristics. The PSO-OPF is successfully and effectively implemented to find the global or quasi-global optimum of the OPF problems. The results show that the optimal dispatch solutions determined by PSO lead to lower active power loss then that found by other methods, which confirms that the PSO is well capable of determining the global or near global optimum dispatch solution. Major drawback of PSO, like in other heuristic optimization techniques, is that it lacks somewhat a solid mathematical foundation for analysis to be overcome in the future, development of relevant theories. And also a relatively longer computation time than mathematical approaches.

RASOUL et al [5] introduces, a new PSO-based algorithm to solve Economic Dispatch (ED) problem of a power system. The solution process is tested on four different case studies from 3 generation unit to 40 generation unit. Their results show improvements in the quality of solution which gives a better result in terms of cost issues. This fact proves that the proposed algorithm has more ability to solve both small and large case studies compared to the existing methods while the solution considers.

Singh et.al [6] carries out a test on economic load dispatch on three and six generator system using PSO algorithm. A comparative analysis is made on the Total generation cost using three methods viz conventional, GA and PSO. It is concluded that PSO algorithm has better ability to save total cost and computational time compared to conventional methods and GA. R. Vijay [7] presents Bacterial Foraging optimization (BFO) to solve problem in power systems. The algorithm is based on the group foraging behavior of Escherichia coli (E-Coli) bacteria present in human intestine. This social foraging behavior of
E.coli bacteria has been used to solve optimization problems. The proposed algorithm has been tested for a test system with 3 and 13 generating units and the results thus obtained are compared with the results of earlier methods (PSO and GA). As compared to other two, the BFO is easy to implement and there are few parameters to adjust. Therefore, BFO has been successfully applied in many areas of power system. From the outcome of the results, it is shown that the proposed algorithm is very effective in giving quality solutions for ELD problems. Moreover, it also reveals that the fuel costs are reduced.

Naveen kumar et al. [3] presents, the application of genetic algorithm to solve the economic load dispatch problem. An analysis is made by applying the GA on two different test systems. The results of the test system -1 using GA are compared with Lambda iteration method (LIM) which shows better results than LIM method and satisfy ELD requirements. A short term optimal generation scheduling for the test system-2 has been obtained. GA is better than the conventional methods from computational point of view.

Nanda et al. presents a paper that demonstrates with clarity, chronological development and successful application of GA to the solution of ELD with Line flow constraints. Two test systems (IEEE 14 bus and 30 bus) have been tested and the ELD results with GA have been compared with those obtained through Classical technique, Linear Programming technique and Quadratic Programming technique. Investigations provide information about the sensitivity of the overall minimum cost to changes in GA parameters. Results also reveal that the algorithm developed for GA works satisfactorily for different magnitudes of Line flow constraints.

Nithiyananthan et. al presents a GA solution to the OPF problem has been presented and applied to small and medium size systems. The main advantage of the GA solution to the OPF problem is its modeling flexibility; non convex unit cost functions, prohibited unit operating zones, discrete and control variables and complex non linear constrains can be easily model. Another advantage is that can be easily coded to work on parallel computers.

Salmon et. al. This paper proposes a Load Flow calculation methodology based on the Particle Swarm Optimization technique. The proposed algorithm was tested for the IEEE 6bus system through the implemented computational program. The Particle Swarm technique main advantages are the flexibility of modeling and its better convergence. The numeric simulations results imply that the proposed algorithm presents better results for the buses power mismatches than the proposed algorithm in and the results are also as good as those obtained through Newton- Raphson method

Ahmed and Singh et al. presents proposed algorithm has better ability to save total cost and computational time as compare to other conventional methods. For large number of generating units the proposed PSO method works better than the conventional methods by reducing computational time. Genetic algorithm suffers for pre mature convergence. Simulation was carried out with three generator and six generator system and analyzed and seen that the total cost was also reduced. It was concluded that the proposed method is simple concept easier to implement is robust to control the parameter and computational 
efficiency is better compared to mathematical algorithm and other optimization techniques such as GA.

\section{CONCLUSIONS}

The discussion shows the different methods involved in solving the nonlinear load flow problems. Further research work can be done for finding more powerful methods to solve the power flow equations with more efficiency in terms of time, computer memory storage as well as robustness. This paper presents important features of Load flow problem. This problem is a traditional problem and solved using several methods based on the requirements of the problem formulation. These methods are classified into mathematical programming based methods, Artificial Intelligence (AI) techniques and hybrid methods. This paper will be helpful for all those who do research related to Optimum load flow solution problem. Some advance AI technique based optimal solution such as GA, PSO and BFO are compared and reviews from different papers were taken. It can be concluded that the PSO and BFO based techniques seems to be faster and more advance method for finding optimum load flow solutions.

\section{REFERENCES}

[1.] Rasoul Rahmani, Mohd Fauzi Othman, Rubiyah Yusof, Marzuki Khalid "Solving economic dispatch problem using particle swarm optimization by an evolutionary technique for initializing particles" Journal of Theoretical and Applied Information Technology. Vol. 46 No.2 pp 526-536 December 2012

[2]. Kwang Y. Lee, Jong-Bae Park, "Application of Particle Swarm Optimization to Economic Dispatch Problem: Advantages and Disadvantages" 2006 IEEE pp 188-193.

[3]. Naveen Kumar, K.P.Singh Parmar, Surender Dahiya "A Genetic Algorithm Approach for the Solution of Economic Load Dispatch Problem" International Journal on Computer Science and Engineering (IJCSE), Vol. 4 No. 06 June 2012 pp 106-1068.
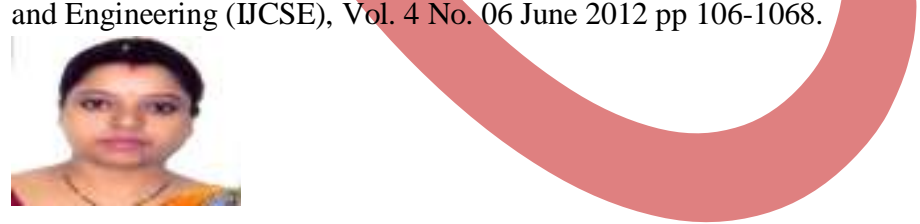

Debirupa Hore was born in Guwahati Assam India on April $19^{\text {th }}$ 1983.She received her B.E Degree in Electrical Engg. from Assam Engg.College Guwahati in 2006 and M.Tech in Energy and Power Systems in 2010 from NIT Silchar. She worked in GIMT Guwahati for 5 years as Assistant Professor. Currently she is working in KJ Educational Institutes, KJCOEMR ,Pune (Maharashtra).Her research areas of interest includes Power Systems, AI Techniques, Power Electronics and Drives.
[4]. J. Nanda*, R. Badri Narayanan "Application of genetic algorithm to economic load dispatch with Lineflow constraints" November 2001 pp 723-729

[5].Amita Mahor, Vishnu Prasad, Saroj Rangnekar "Economic dispatch using particle swarm optimization: A review" Renewable and Sustainable Energy Reviews 13 (2009) pp 2134 2141.

[6]. Rajesh K.S. Prof. S. Lyla, Prof. Bisharathu Beevi "Economic Load Dispatch based on Genetic Algorithm"

[7]. K. Nithiyananthan, V. Ramachandran" Enhanced Genetic Algorithm Based Model for Power System”Vol I June 2011.

[8]. R. Vijay "Intelligent Bacterial Foraging Optimization Technique to Economic Load Dispatch Problem" International Journal of Soft Computing and Engineering (IJSCE) ISSN: 2231-2307, Volume-2, Issue-2, May 2012.

[9]. P. S. Bhowmik, D. V. Rajan, S. P. Bose "Load Flow Analysis: An Overview" World Academy of Science, Engineering and Technology, 2012.

[10]. Salomon, Torres, Martins, "Load Flow Computation via Particle Swarm Optimization" ISEE Electrical Systems and Energy Institute.

[11]. Satyendra Singh, K.S. Verma" Optimal Power Flow using Genetic Algorithm andParticle Swarm Optimization" Vol. 2 Issue 1, Jan.2012, pp. 046-049.

[12]. Djillani BEN ATTOUS, Yacine LABBI" Particle Swarm Optimization based Optimal Power Flow for Units with NonSmooth Fuel Cost Functions" El-Oued University Center, Algeria. pp 377-381.

[13].Anish Ahmed, Nitin Singh, Tarun Varshney "A New Approach for solving economic load dispatch problem", MIT International journal of Electrical and instrumentation Engineering, Vol 1 Aug 2011 pp 93-98.

[14]. Dong Hwa Kim , Ajith Abraham „, Jae Hoon Cho” A hybrid genetic algorithm and bacterial foraging approach For global optimization"Publisher Elsevier 2007 pp 3918-3937.

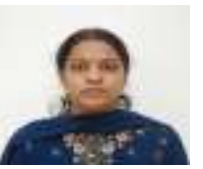

Netra Lokhande received her B.E Degree in Electrical and Electronics Engg. from Karnatak University in 1997 and M.E in Power Systems in 2004 from Government College of Engg.Pune(Pune University). She is currently pursuing Ph.D in Image Processing. She is having around 15 years of Teaching Experience as Assistant Professor. Currently she is working in KJ Educational Institutes, KJCOEMR, Pune(Maharashtra).as Head of Department. Her research areas of interest includes Power Systems, AI Techniques, Power Electronics and Drives, Image Processing. 